\title{
Evaluation of Control Point Selection in Automatic, Mutual Information Driven, 3D Warping
}

\author{
Chuck Meyer, Jennifer Boes, Boklye Kim, and Peyton Bland \\ Department of Radiology, University of Michigan, Ann Arbor, MI 48109-0553 \\ cmeyer@umich.edu \\ http://www.med.umich.edu/dipl
}

This work was supported in part by DHHS PHS NIH 1R01 CA59412

\begin{abstract}
We have implemented automatic 3D thin-plate spline warping as a geometric interpolant to map one dataset volume onto another. Homologous control points in one space are iteratively moved by an optimizer to maximize the global mutual information between the two data volumes. Given two different poses between highly deformed objects we desire to compute the relative geometric deformation using a minimal set of control points as determined by number and placement. The general solution to this problem is not known. In this paper we assess retrospective control point selection for the case of significant patient motion during MRI breast imaging.
\end{abstract}

\section{Introduction}

Optimization of mutual information (MI) to drive the automatic affine registration of multimodality data volume sets has been actively pursued for the last 3-4 years [1-6]. Over the last 2 year period we have demonstrated that MI can be used to drive automatic thin-plate spline (TPS) warping as well [7-10]. The process is implemented by allowing an optimizer, the Nelder-Mead simplex algorithm in this case, to drive the positions of homologous control points in the homologous data set, i.e. the data volume to be mapped onto the reference volume, subject to maximizing the resultant MI between the reference volume and the transformed homologous volume.

For cases where the bending energy of the TPS warping is small [11], nearly any evenly distributed, volumetric placement of a few control points, e.g. more than four and fewer than 10 , in the reference volume is sufficient to compute a good registration. In our typical implementation only the first 3 homologous control point pairs must be initially placed in the homologous volume. Then the 6 degree of freedom (DOF) rigid body registration is computed by the optimizer's movement of the 3 control points in homologous space to maximize MI. Next, using the optimized rigid body model, the first 4 control points in the reference space are mapped into the homologous space, and then the $12 \mathrm{DOF}$, full affine registration is subsequently computed, again by optimizing MI through iterative movement of the 4 control points 
in homologous space. Finally the optimized warping solution is initiated by using the previously optimized full affine solution to map all of the reference control points into the homologous volume.

However, in cases involving significant deformation between initial poses of the data sets, our typical implementation described above may fail. For these problematic circumstances we desire to know the minimal number of homologous control point pairs and the range of their initial placement to subsequently converge to a good geometric model of the warping between the two poses. The remainder of this paper examines criteria for the retrospective evaluation of control point pairs in the situation where the DOF of the warping is initially overdetermined, i.e. more than the necessary number of homologous control point pairs are initially chosen to determine the warp. The physical data set and registered results appear in the latter part of this paper.

\section{Methods}

Although the algorithm, mutual information for multimodality image fusion (MIAMI Fuse), was developed for multimodality registration, in this case it has been applied to two MRI breast volumes acquired before and after significant patient movement. A case for the use of a multimodality registration algorithm can be made by noting that tissue intensities can vary dramatically depending on changes in tissue positioning with respect to breast coil location, i.e. B1-field inhomogeneitities. Repositioning of the breast occurs with patient movements during a single exam, or more obviously when exams are repeated at 6-12 week intervals. Volume imaging data were acquired in coronal planes over both breasts of the patient lying prone over breast coil wells inside the $1.5 \mathrm{~T}$ magnet. Voxel dimensions were $1.18 \times 1.18 \times 5.5$ $\mathrm{mm}^{3}$. Since only the patient's left breast was significantly deformed, 18 nearly homologous control points in the left breast were identified manually in both the pre and post movement data volume. Using these points as the initial starting vector for automatic registration via TPS warping resulted in an initial MI of 1.01 bits, where the entropy in the reference data set was 4.576 bits. The optimization algorithm was repeatedly run from start to finish 8 times using random control point placements up to $3 \mathrm{~mm}$ city block metric from the manually chosen starting vector. Each optimization "run" consists of many repeated optimization cycles. Each cycle consists of a single decent to the cost function, i.e. -MI, minimum, where the minimum was detected when the optimizer called for all control points to move less than $0.5 \mathrm{~mm}$ in any coordinate axis direction from the previous iteration. The process of repeating optimization cycles stopped (which defined a "run") when the optimized cycle value of MI changed less than 0.0001 bit over 3 previous optimization cycles. This process of repeating optimizations from starting vectors randomly distributed around the previous cycle's solution is used to prevent entrapment by a local minimum. 


\section{Results}

As averaged over the 8 runs, the number of times the objective function was evaluated for each run was 12271 (sem = 1331). Each full run required an average of 2 hours on a $433 \mathrm{MHz}$ DEC Alpha personal workstation running Digital UNIX V4.0C. Figure 1 describes the typical behavior of the cost function vs. number of iterations.

The variance of each control point's stopping position in each of the coordinate directions was computed and compared to the second partial derivative of the cost function in the same direction at one of the computed solutions for the same control point. Figure 2 illustrates the excellent inverse correlation between the components of the second partials and variance in the final control point positions.

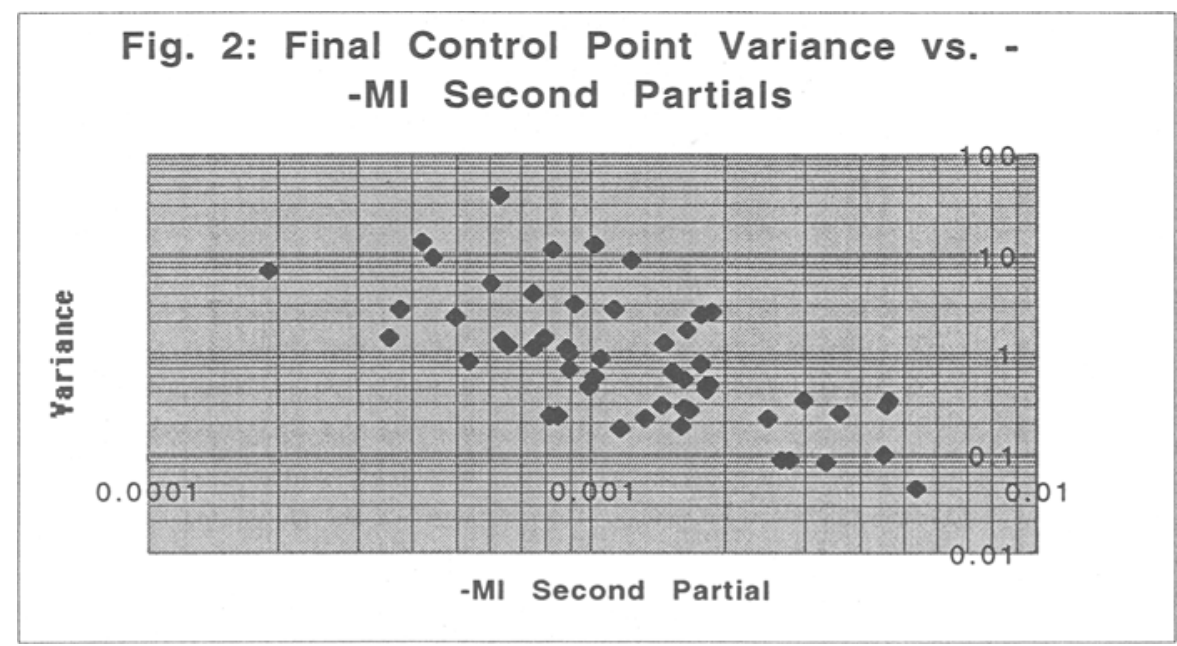

After removing the control point with the most variance, i.e. rms error for all three vector components, 8 more optimized registrations were completed. The same process was repeated using 16 and 10 control points where the points removed ranked highest in variance. In the original set of 18 control points, the standard error around the average solution for one (outlier) control point was more than 3 times that of the average, while two points were more than 2 times the average. In the distribution of final control point positions for the set of 17, all points had standard errors less than 2 times the average. Figure 3 summarizes the generalized dependence of the final cost function, -MI, vs. number of control points or DOF. Although only the results 
for $\leq 16$ control points are significantly different from the result using 18 points, there appears to be a smooth trend towards lesser MI values as the number of control points decrease, only .

Finally in an effort to prospectively evaluate which of the set of proposed control points might be the most valuable toward computing a good solution, we examined the initial gradient magnitude of $\mathrm{MI}$ for each control point at the manually selected starting vector.

Fig. 4 illustrates the results of this experiment. Note that choosing control points according to rank by MI gradient magnitude at the start vector removes valuable points that had some of the smaller variances around the final solution. The same lack of correlation is
Fig. 3: Optimized -MI vs. Number of Control Points

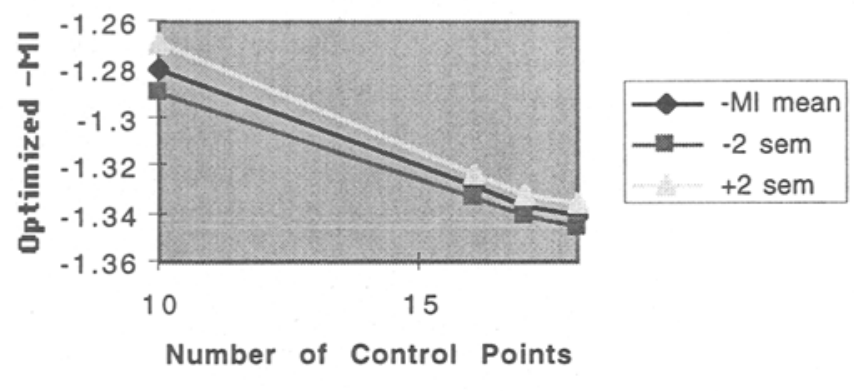

Fig. 4: Final Control Point RMS Error

vs. Initial MI Gradient Magnitude

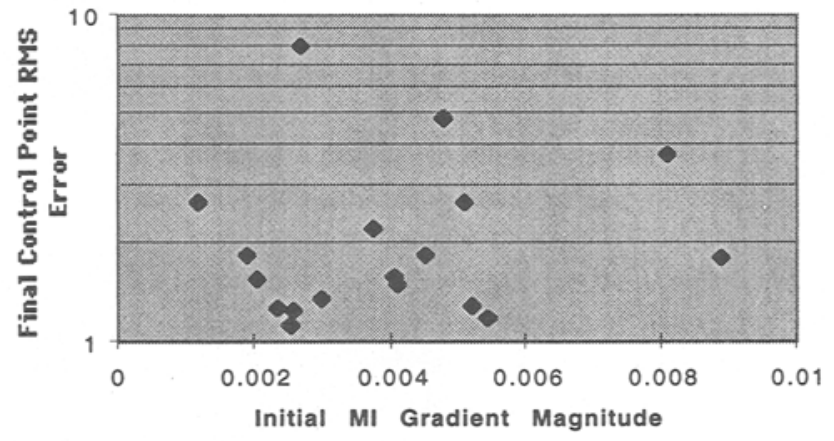

Fig. 5: Final Del Squared MI vs. Initial Gradient Magnitude

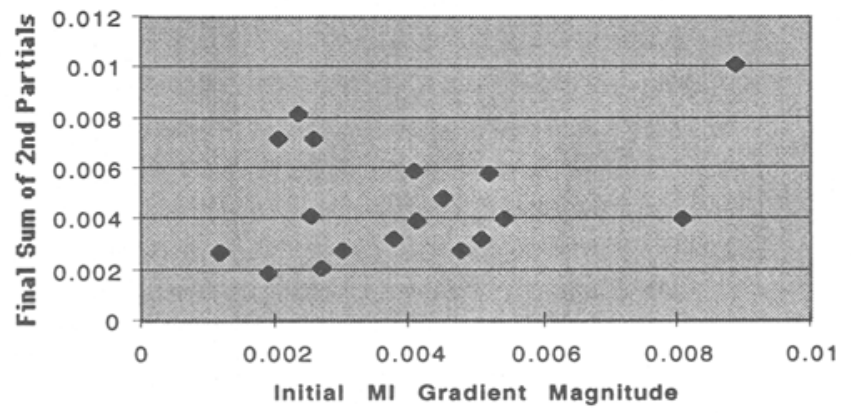


observed between initial gradient magnitude and the sum of the second partial derivative components as shown in Fig. 5.

The general problem is better visually illustrated by selecting the appropriate slices in the pre- and postmotion data sets. The presence of warping between the 2 poses is clearly shown in Fig. 6 for the patient's left breast (seen on the reader's right, i.e. reader is vicwing the patient from the front). Although not demonstrated here, the right breast was much less deformed by the movement.

Fig. 7 displays the geometric warping computed to warp the postmotion data onto the premotion data using a gridded cube. Onc plane of the registered volume is shown in its correct position within the deformation grid (the viewer is now positioned behind the patient). The deformation was computed using 18 control points for the left breast.

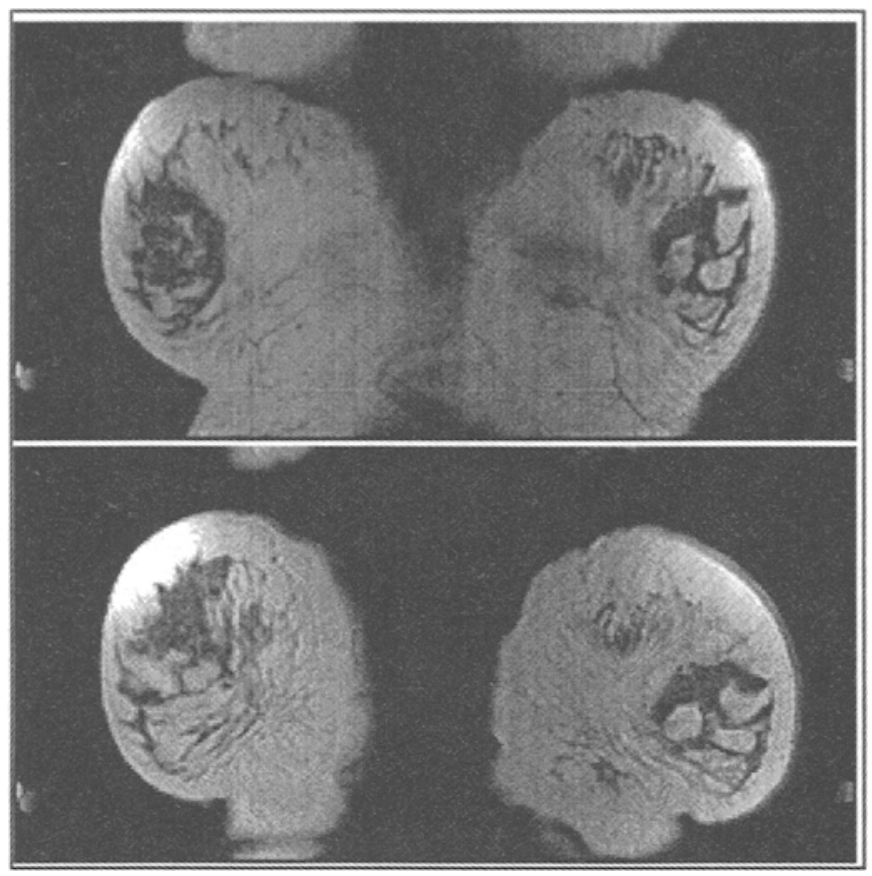

Figure 6. Note significant deformation in left breast ( $\uparrow$ ) before (upper) and after (lower) patient repositioning.

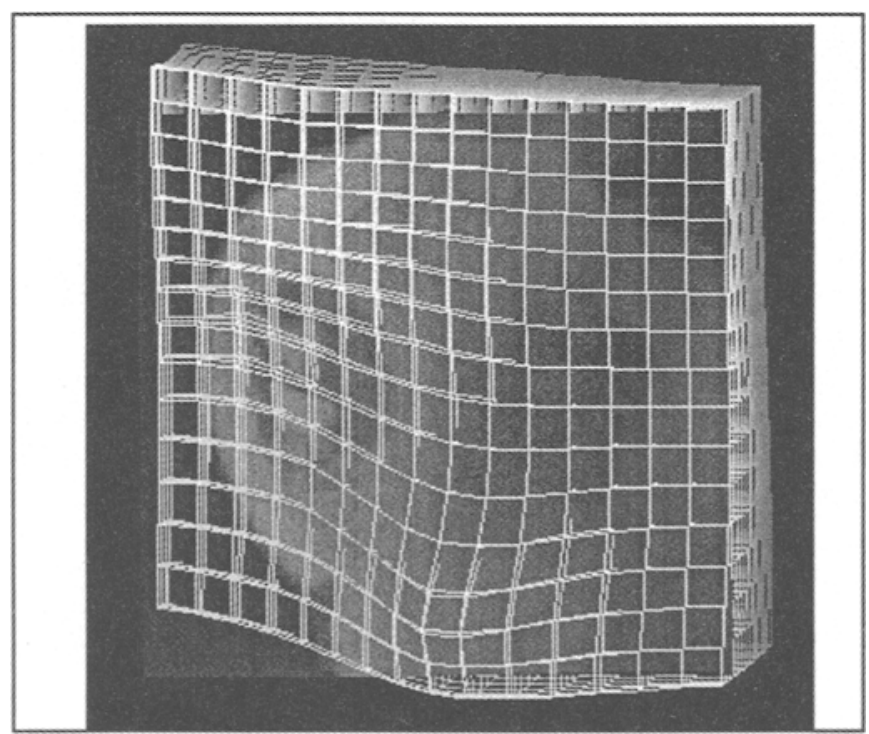

Figure 7. Volumetric deformation required to remove motion. 


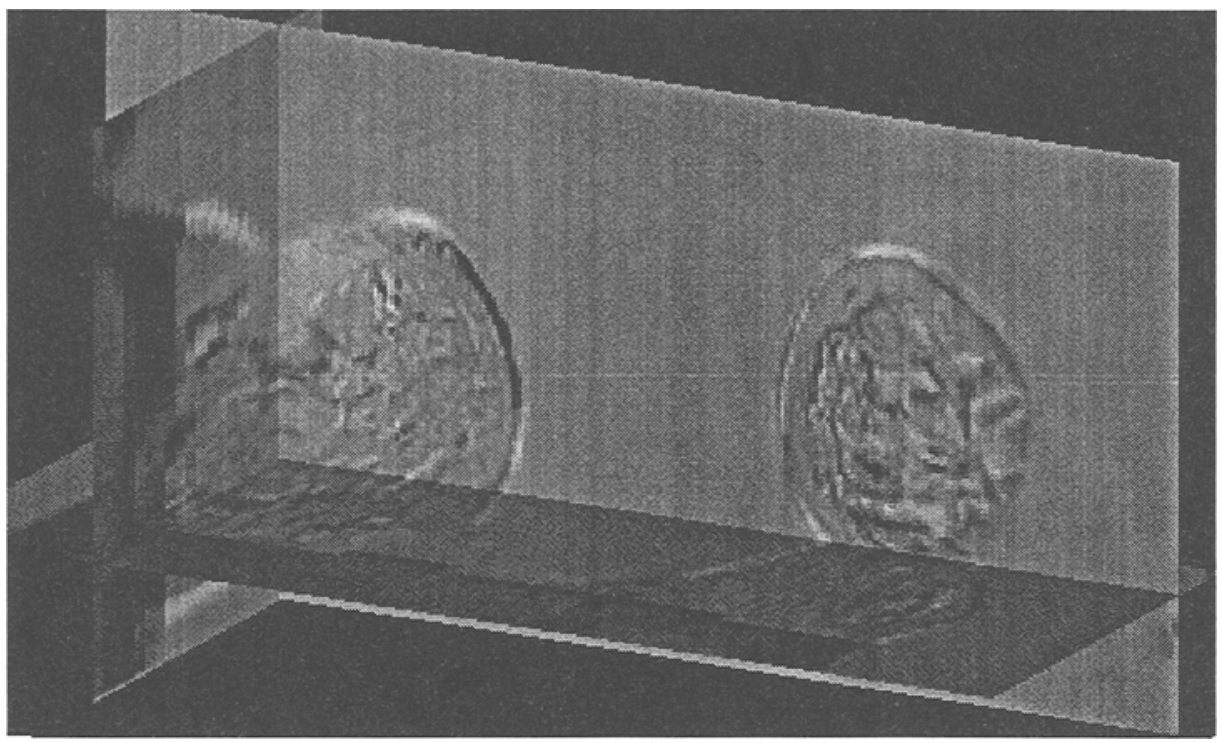

Figure 8. Difference volume obtained after mapping postmotion onto premotion data volume, where mapping was computed using 24 total control points.

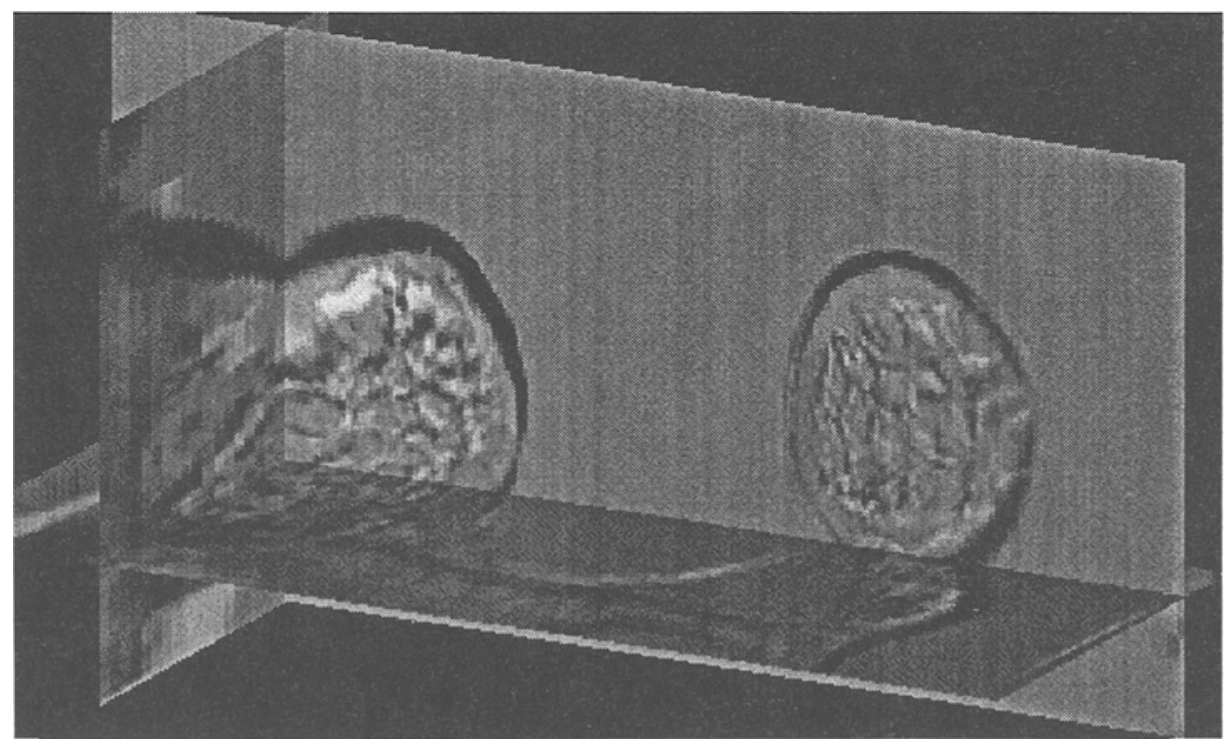

Figure 9. Difference volume obtained after mapping postmotion onto premotion data volume, where mapping was computed using 8 total control points. 
Figs 8 and 9 demonstrate the improved spatial registration associated with the increase in number of effective control points or DOF. In Figs. $8 \& 9$ black-to-white represents a difference range from -128 to +128 , respectively; perfect registration would be indicated by midscale gray corresponding to a difference of nearly zero. Essentially the same information is conveyed by Fig. 3 in terms of increasing MI (decreasing -MI) as a function of number of control points, although the consequences are a little less visual. The use of a 6 control point, TPS warping was sufficient to register the right breast (seen in Fig. 9 on the reader's right as viewed from behind the patient). The combined registration was obtained using the separately computed, optimized positions for the 6 control points of the right breast and 18 control points of the left. A single 24 homologous point, TPS warping was computed to register the pre and post motion data sets. Data missing from the warped data set can be seen on the axial and sagittal planes in Fig. 8, where the mapping called for data that was outside the field of view of the postmotion data volume, and was thus rendered as zeros. In such cases of limited fields of view it is important not to penalize the resultant MI for the missing data that maps outside of the reference volume.

\section{Conclusions}

When the information content of the data volumes is sufficient, reliable TPS warped registrations can be routinely obtained, even in poses containing high bending energy. The minimal subset, i.e. number and placement, of control points in the reference and homologous data volumes is generally unknown to generate an acceptable solution as defined by a minimal mutual information criterion. In a retrospective evaluation of an overdetermined set of manually selected control points we found good inverse agreement between the curvature of the cost function, i.e. -MI, and the variance in the final positions of the control points. The greater the curvature of the cost function at its minimum with respect to movement of a control point in any one direction, the smaller the variance in the final resting position along that direction over repeated random trials. However, prospective evaluation of the local gradient magnitude of the cost function at potential, initial starting positions of the control functions was seemingly uncorrelated with the warping value of the control point as judged by either its variance or curvature at the locus of the final solution position.

Clearly it is desirable to eliminate candidate control points from an automatic instantiation of an overdetermined grid of possible starting points. Just as clearly it is possible to eliminate some control points where one of the pairs lies centrally in a relatively large region of no texture where the optimizer can radically reposition the control point while generating large bending energies with little or no change in the MI cost function. However sorting of control points based on their registration value in computing the desired warping will likely require more than estimates of local MI gradients. 


\section{References}

[1] A. Collignon, F. Maes, D. Delaere, D. Vandermeulen, P. Suetens, and G. Marchal, "Automated multimodality image registration using information theory," presented at Computational Imaging and Vision, Ile de Berder, FR, 1995.

[2] D. L. G. Hill, C. Studholme, and D. J. Hawkes, "Voxel similarity measures for automated image registration," presented at Visualization in Biomedical Computing, Rochester, MN, 1994.

[3] C. Studholme, D. Hill, and D. Hawkes, "Multiresolution voxel similarity measures for MR-PET registration," presented at Computational Imaging and Vision, lle de Berder, FR, 1995.

[4] F. Maes, A. Collignon, D. Vandermeulen, G. Marchal, and P. Suetens, "Multimodality image registration by maximization of mutual information," IEEE Transactions on Medical Imaging, vol. 16, pp. 187-198, 1997.

[5] P. Viola and W. M. Wells, "Alignment by maximization of mutual information," presented at 5th Int'l. Conf. on Computer Vision, MIT, 1995.

[6] W. Wells, P. Viola, and R. Kikinis, "Multimodal volume registration by maximization of mutual information," presented at Medical Robotics and Computer Assisted Surgery II, Philadelphia, 1995.

[7] B. Kim, J. L. Boes, K. A. Frey, and C. R. Meyer, "Mutual information for automated multimodal image warping," presented at Visualization in Biomedical Computing, Hamburg, Germany, 1996.

[8] B. Kim, J. L. Boes, K. A. Frey, and C. R. Meyer, "Mutual information for automated unwarping of rat brain autoradiographs," Neurolmage, vol. 5, pp. 3140, 1997.

[9] C. R. Meyer, J. L. Boes, B. Kim, P. H. Bland, and K. A. Frey, "Warping normal patients onto the ICBM atlas by maximizing MI," presented at 4th Int'l Conference of Functional Mapping of the Human Brain, Montreal, Quebec, CA, 1998.

[10] C. R. Meyer, J. L. Boes, B. Kim, P. Bland, K. R. Zasadny, P. V. Kison, K. Koral, K. A. Frey, and R. L. Wahl, "Demonstration of accuracy and clinical versatility of mutual information for automatic multimodality image fusion using affine and thin plate spline warped geometric deformations," Medical Image Analysis, vol. 3, pp. 195-206, 1997.

[11] F. L. Bookstein, Morphometric tools for landmark data: geometry and biology. Cambridge: Cambridge University Press, 1991. 